gr-qc/9212007

\title{
Weak-Field Gravity of Revolving Circular Cosmic Strings
}

\author{
*Des J. Mc Manus \\ School of Theoretical Physics, Dublin Institute for Advanced Studies, \\ 10 Burlington Road, Dublin 4, Ireland. \\ $\dagger$ Michel A. Vandyck \\ Physics Department, University College Cork, Ireland, \\ and \\ Physics Department, Cork Regional Technical College, \\ Bishopstown, Co. Cork, Ireland.
}

\begin{abstract}
A weak-field solution of Einstein's equations is constructed. It is generated by a circular cosmic string revolving in its plane about the centre of the circle. (The revolution is introduced to prevent the string from collapsing.) This solution exhibits a conical singularity, and the corresponding deficit angle is the same as for a straight string of the same linear energy density, irrespective of the angular velocity of the string.
\end{abstract}

PACS numbers: 04.20-q, 98.90+s

To appear in Physical Review D 


\section{Introduction}

One of the most notable features of a straight cosmic string [1] is the presence in spacetime of an angular deficit, the magnitude of which is related to the linear energy density $\mu$ of the string by $\delta \psi=8 \pi G \mu$. Indeed, the deficit-angle structure of spacetime is central to many proposals for the possible observation of the gravitational effects of cosmic strings [2].

The deficit-angle model is generally accepted as a good approximation for describing the exterior gravitational field of cosmic strings. Frolov, Israel, and Unruh (FIU) used precisely this approximation when they considered a thin circular string at a moment of time symmetry [3].

Recently, we investigated further [4] the problem of the deficit angle produced by circular strings. We constructed a weak-field stationary solution of Einstein's equations generated by a thin circular string and established that external radial stresses had to be introduced to support the ring against collapse (thus allowing a stationary solution to exist). The form of the radial stresses was completely determined by stress-energy conservation. The main result of our study was that a circular string produces conical singularities with the same angular deficit as a straight string of identical linear energy density, fully supporting FIU's assumption. Thus, we demonstrated, in the weak-field limit, the validity of the FIU hypothesis directly from the field equations. Furthermore, the external radial stresses were seen not to contribute to this angular deficit.

In the present work, we ask the question whether it is possible to extend the previous results to a self-supporting circular string, as opposed to an externally supported circular string: can the stabilising role previously played by external radial stresses be played by an internal mechanism? Centrifugal force is the simplest candidate; it should be possible to prevent gravitational collapse by spinning the ring at an appropriate angular velocity.

We construct a weak-field solution of Einstein's equations corresponding to an infinitely thin circular string revolving at a given angular velocity $\omega$. We begin by examining the most general scenario where the string is partially supported by centrifugal force and partially by external radial stresses. The angular velocity is chosen arbitrarily and the radial stresses are then determined by stress-energy conservation. The angular deficit produced by this solution is found to be equal to the deficit produced by a straight string (of the same linear energy density), irrespective of the value of the angular velocity $\omega$ (within the limitation of the weak-field approximation). Later, we also calculate and discuss the critical angular velocity $\omega_{\text {crit }}$ at which the ring is totally self-supporting, namely the particular velocity at which no radial stresses are necessary to support the ring. The latter is then supported entirely by centrifugal force. 
In this paper, we use units in which $\hbar=c=1$, take the metric to have signature $(-,+,+,+)$ and adopt the geometrical conventions of Synge [5].

\section{The Stress-Energy Tensor}

The stress-energy tensor $T^{\mu}{ }_{\nu}$ generating the gravitational field of a revolving string partially supported against collapse by external radial stresses contains three contributions: ${ }^{M} T^{\mu}{ }_{\nu}$, the contribution from the circular motion of the ring (excluding radial stresses required to maintain circular motion), ${ }^{A} T^{\mu}{ }_{\nu}$, the contribution from the azimuthal flux through the string (which corresponds to the $T^{z}{ }_{z}$ stresses for Vilenkin's straight string), and ${ }^{E} T_{\nu}^{\mu}$, the contribution from the external radial stresses. Given that the diameter of the core of a string arising from a spontaneously broken gauge theory [6] is microscopically small, it is well justified to make the approximation that the stress-energy tensors ${ }^{M} T^{\mu}{ }_{\nu}$ and ${ }^{A} T^{\mu}{ }_{\nu}$ of the circular string be confined to the infinitely thin ring $r=a, z=0$, where $a$ denotes the radius of the ring. On the other hand, the radial stresses ${ }^{E} T^{\mu}{ }_{\nu}$, being external, are not confined to the core of the string. (See [1] for a more detailed discussion.)

For (pressure-free) dust of rest-energy density $\rho_{0}$, moving with four-velocity $u^{\mu}$, the stress-energy tensor is ${ }^{M} T_{\nu}^{\mu}=\rho_{0} u^{\mu} u_{\nu}$, where $u^{\mu} u_{\mu}=-1$. In cylindrical coordinates $x^{\mu} \equiv(t, \phi, r, z)$, the four-velocity $u^{\mu}$ of an infinitely thin ring of radius $a$, centered at the origin, lying in the $x-y$ plane, and revolving with angular velocity $\omega$ is readily found as

$$
\begin{aligned}
u^{\mu} & =\Gamma\left(\delta_{t}^{\mu}+\omega \delta_{\phi}^{\mu}\right) \\
-\Gamma^{-2} & \equiv g_{t t}(a, 0)+2 \omega g_{t \phi}(a, 0)+\omega^{2} g_{\phi \phi}(a, 0)
\end{aligned}
$$

where $g_{\mu \nu}(a, 0)$ denotes the metric components evaluated at $r=a, z=0$. (The metric enters this equation because of the normalisation condition $u^{\nu} u_{\nu}=-1$.)

Furthermore, the ring has its rest-energy density $\rho_{0}$ purely localised:

$$
\rho_{0}=\mu_{0} \delta(r-a) \delta(z)
$$

where $\mu_{0}$ is the linear rest-energy density. Consequently, the stress-energy tensor ${ }^{M} T^{\mu}{ }_{\nu}$ produced by the circular motion has the expression

$$
{ }^{M} T^{\mu}{ }_{\nu}=\mu_{0} \Gamma^{2}\left(\delta_{t}^{\mu}+\omega \delta_{\phi}^{\mu}\right)\left[g_{\nu t}(a, 0)+\omega g_{\nu \phi}(a, 0)\right] \delta(r-a) \delta(z),
$$

where $g$ is the metric and no summation is implied over repeated indices $t$ and $\phi$. (The summation convention will be suspended all throughout when $t, \phi$ or $r$ appear as subscripts or superscripts.) 
The stress-energy tensor giving the contribution from the azimuthal flux through the string is elementary in the case of a non-revolving ring [4], the simple argument being that ${ }^{A} T^{\phi}{ }_{\phi}$ plays the role that $T^{z}{ }_{z}$ plays for a straight string. However, a more detailed reasoning is necessary when the string is revolving since a revolving frame is not inertial. The appropriate stress-energy tensor ${ }^{A} T^{\mu}{ }_{\nu}$ can be established by employing the general method applicable to anisotropic fluids [5]. (The "fluid" must be anisotropic since for physical reasons we expect a pressure along the $\phi$ axis but none along the $r$ and $z$ axes.)

The stress-energy tensor [5] of a fluid of rest-energy density $\rho_{0}$, moving with a fourvelocity $u^{\mu}$, is

$$
T^{\mu}{ }_{\nu}=\rho_{0} u^{\mu} u_{\nu}-S^{\mu}{ }_{\nu}
$$

in which the tensor $S^{\mu}{ }_{\nu}$, the stress tensor, satisfies $S^{\mu}{ }_{\nu} u^{\nu}=0$. The eigenvectors $\lambda_{(i)}^{\mu}$, assumed space-like, and the corresponding eigenvalues $-P_{(i)}, 1 \leq i \leq 3$ are called respectively the "principal axes" and the "principal stresses" of $S^{\mu}{ }_{\nu}$. In terms of these quantities, the stress tensor can be expressed as

$$
S_{\nu}^{\mu}=-\sum_{i=1}^{3} P_{(i)} \lambda_{(i)}^{\mu} \lambda_{(i) \nu}
$$

where $\lambda_{(i)}^{\mu}$ satisfies

$$
\begin{aligned}
u_{\mu} \lambda_{(i)}^{\mu} & =0 \\
\lambda_{(i)}^{\mu} \lambda_{(j) \mu} & =\delta_{i j} .
\end{aligned}
$$

The stress-energy tensor ${ }^{M} T^{\mu}{ }_{\nu}$ of (2.4) already takes into account the motion of the ring; therefore, we only consider the stress part $S^{\mu}{ }_{\nu}$ of (2.5). The physical requirement that there be no pressure along the $r$ and $z$ axes implies that the principal pressures $P_{(2)}$ and $P_{(3)}$ vanish. Thus, the only non-zero principal pressure is the azimuthal pressure $P_{(1)} \equiv P_{(\phi)}$. For an infinitely thin ring, the azimuthal pressure is confined to the core of the string, which implies

$$
P_{(1)}=k_{0} \delta(r-a) \delta(z),
$$

where $k_{0}$ is a constant with dimensions of force. (We interpret $-k_{0}$ as the tension in the ring.)

Moreover, the only relevant principal axis, $\lambda_{(1)}$, must point spatially along the $\phi$ direction. The constraint (2.8), together with (2.7) applied to the velocity (2.1), determines $\lambda_{(1)}$ as

$$
\lambda_{(1)}^{\mu}=K \delta_{t}^{\mu}+L \delta_{\phi}^{\mu}
$$


where $K$ and $L$ are solutions of the system

$$
\begin{aligned}
K g_{t t}(a, 0)+(L+\omega K) g_{t \phi}(a, 0)+\omega L g_{\phi \phi}(a, 0) & =0 \\
K^{2} g_{t t}(a, 0)+2 K L g_{t \phi}(a, 0)+L^{2} g_{\phi \phi}(a, 0) & =1 .
\end{aligned}
$$

Thus, we reach the conclusion that the stress-energy tensor ${ }^{A} T^{\mu}{ }_{\nu}$ generated by the azimuthal flux along the string reads

$$
{ }^{A} T_{\nu}^{\mu}=k_{0}\left(K \delta_{t}^{\mu}+L \delta_{\phi}^{\mu}\right)\left[K g_{\nu t}(a, 0)+L g_{\nu \phi}(a, 0)\right] \delta(r-a) \delta(z)
$$

(with no summation on $t$ or $\phi$ ). This tensor depends on two scalar parameters: $\omega$, the angular velocity of the ring, and $-k_{0}$, the tension in the ring. When $\omega$ vanishes, (2.13) becomes identical to the expression obtained in the non-revolving case [4], so that it is consistent to identify the scalar $k_{0}$ with the parameter $k$ in 4 . Consequently, for string matter, it is reasonable to generalise the equation of state $k=-\mu$ of the non-revolving case as $k_{0}=-\mu_{0}$ in the revolving case.

In order to support the ring partially by external radial stresses, we assume the

existence of a radial component of ${ }^{E} T^{\mu}{ }_{\nu}$ confined to the $x-y$ plane (as in the case [由] of a non-revolving ring):

$$
{ }^{E} T_{\nu}^{\mu} \equiv \Delta(r, z) \delta_{r}^{\mu} \delta_{\nu}^{r} \equiv f(r) \delta(z) \delta_{r}^{\mu} \delta_{\nu}^{r}
$$

where $f$ is a function to be determined later by stress-energy conservation, and there is no summation over $r$.

The complete stress-energy tensor $T^{\mu}{ }_{\nu}$ generating the gravitational field of the revolving string is the sum of all the above contributions (2.4), (2.13), and (2.14):

$$
T_{\nu}^{\mu}={ }^{M} T_{\nu}^{\mu}+{ }^{A} T_{\nu}^{\mu}+{ }^{E} T_{\nu}^{\mu}
$$

We now turn to the form of the spacetime metric $g$.

\section{The Metric and Field Equations}

The most general stationary metric produced by an axially-symmetric revolving source may be written [7]

$$
d s^{2}=-e^{2 \nu} d t^{2}+e^{2 \zeta-2 \nu} r^{2}(d \phi-A d t)^{2}+e^{2 \eta-2 \nu}\left(d r^{2}+d z^{2}\right)
$$

where $\nu, \zeta, \eta$, and $A$ are functions of $r$ and $z$ only. 
We proceed as in the non-revolving case [4] and make the weak-field approximation. Thus, we calculate the Einstein tensor $G^{\mu}{ }_{\nu}$ [7] for the metric (3.1) and retain only first-order terms in $\nu, \eta, \zeta$, and $A$.

Given that $A$ is not dimensionless, it is not immediately apparent that terms of order $A^{2}$ may be neglected. However, for the stress-energy tensor (2.4), (2.13)-(2.15), the linearised Einstein field equations show that this is indeed the case at first order in the dimensionless quantities $G \mu_{0}$ and $G k_{0}$. (A complete discussion is presented in Appendix A.) Therefore, in all our future considerations, the expression "weak-field approximation" will refer to the expansion of the field equations at first order in $\nu, \eta, \zeta, A, G \mu_{0}$, and $G k_{0}$.

With this approximation, the equations (2.2), (2.4), and (2.11)-(2.13) simplify greatly, and after some manipulations the field equations (see appendix A) reduce to

$$
\begin{aligned}
\nabla^{2} \nu & =4 \pi G\left\{\left(C_{1}+C_{2}\right) \delta(r-a) \delta(z)+\Delta(r, z)\right\} \\
\widetilde{\nabla}^{2} \eta & =8 \pi G C_{2} \delta(r-a) \delta(z) \\
\nabla^{2} \zeta+\frac{1}{r} \zeta_{r} & =8 \pi G \Delta(r, z) \\
\frac{\partial}{\partial r}(r \zeta) & =\eta-\eta_{0} \\
\nabla^{2} A+\frac{2}{r} A_{r} & =-16 \pi G C_{3} a^{-1} \delta(r-a) \delta(z)
\end{aligned}
$$

in which $\Delta$ is the radial-stress function defined in (2.14), $\widetilde{\nabla} \equiv \partial_{r}^{2}+\partial_{z}^{2}, \quad \nabla \equiv \widetilde{\nabla}+(1 / r) \partial_{r}$ is the Laplacian, $\eta_{0}$ is an arbitrary constant of integration, and the constants $C_{i}, 1 \leq i \leq 3$ are related to the parameters $\mu_{0}, k_{0}, \omega$, and $a$ of the problem by

$$
\begin{aligned}
C_{1} & \equiv\left(\mu_{0}+\omega^{2} a^{2} k_{0}\right) \Gamma_{0}^{2} \\
C_{2} & \equiv\left(\omega^{2} a^{2} \mu_{0}+k_{0}\right) \Gamma_{0}^{2} \\
C_{3} & \equiv\left(\mu_{0}+k_{0}\right) \Gamma_{0}^{2} \omega a \\
\Gamma_{0}^{-2} & \equiv 1-\omega^{2} a^{2} .
\end{aligned}
$$

(The quantities $G C_{i}, 1 \leq i \leq 3$, are dimensionless.)

The compatibility condition for (3.3)- 3.5$)$, or equivalently the stress-energy conservation law, determines the radial-stress function $\Delta$ of (2.14) as

$$
\Delta(r, z)=f(r) \delta(z)=\left(C_{2} / r\right) \Theta(r-a) \delta(z)
$$

in which $\Theta$ denotes the Heaviside step function. This expression, which is similar to the one obtained in the non-revolving case [4], may now be substituted into (3.2), so that we have a complete set of equations of which the metric functions $\nu, \zeta, \eta$, and $A$ are solutions. 


\section{Angular Deficit}

Our main purpose in solving the field equations (3.2)-(3.6) is to investigate the metric for conical singularities and to calculate the corresponding angular deficit. Because of the fact that conical singularities involve only the metric (3.1) at constant time $t$ and constant azimuth $\phi$, it is sufficient to restrict attention to obtaining explicitly the functions $\nu$ and $\eta$ given by (3.2) and (3.3). We solved these equations, for different values of the constants $C_{i}$, in our previous work on the non-revolving ring [4], and it is therefore not necessary to repeat the analysis here. We only recall that, in toroidal 8 coordinates $(t, \phi, \sigma, \psi)$, which are related to the cylindrical coordinates $(t, \phi, r, z)$ by

$$
z / a \equiv N^{-2} \sin \psi \quad r / a \equiv N^{-2} \operatorname{sh} \sigma \quad N^{2} \equiv N^{2}(\sigma, \psi) \equiv \operatorname{ch} \sigma-\cos \psi
$$

the solutions near the string (namely for $\sigma \rightarrow \infty$ ) read:

$$
\begin{aligned}
& \nu(\sigma, \psi) \rightarrow-2 G\left(C_{1}+C_{2}\right) \sigma \\
& \eta(\sigma, \psi) \rightarrow-4 G C_{2} \sigma .
\end{aligned}
$$

(As in the non-revolving case [4], the radial stresses (2.14) and (3.11) do not contribute to these asymptotic forms for $\nu$ and $\eta$, and thus have no influence on the conical singularities.)

It follows from (4.2) and (4.3) that the combination $\eta-\nu$, which determines the metric (3.1) at constant $t$ and $\phi$, becomes, after substituting the definitions (3.7), (3.8), (3.10) and noting the non-trivial cancellation of the $\omega$-dependent terms:

$$
\eta-\nu \rightarrow 2 G\left(\mu_{0}-k_{0}\right) \sigma
$$

The fact that $\eta-\nu$ is proportional to the toroidal coordinate $\sigma$ indicates the presence of a conical singularity [3, 4 . The corresponding angular deficit $\delta \psi$, which is related to the ratio of the perimeter of a circle centered at the core of the string to the radius of this circle [3, 4], is given by

$$
\delta \psi=2 \pi-\lim _{\sigma_{0} \rightarrow \infty}\left[\left.\int_{-\pi}^{\pi} d \psi\left(N^{-2} \mathrm{e}^{\eta-\nu}\right)\right|_{\sigma=\sigma_{0}} / \int_{\sigma_{0}}^{\infty} d \sigma N^{-2} \mathrm{e}^{\eta-\nu}\right],
$$

and is easily calculated as

$$
\delta \psi=4 \pi G\left(\mu_{0}-k_{0}\right)
$$

As announced earlier, this expression is independent of the angular velocity $\omega$ at which the ring revolves. Moreover, the angular deficit is also identical with Vilenkin's result for a straight string. (For string matter, $k_{0}=-\mu_{0}$, as explained in [4.) We have thus demonstrated, in the weak-field approximation, that a revolving circular string produces the same angular deficit as a straight string of the same linear energy density. 


\section{Self-supporting Ring}

Finally, we address the problem of whether a revolving string can be totally self-supporting. Up to this point, we considered a ring partially supported by the Centrifugal Force produced by the revolution and partially supported by the external radial stresses $\Delta$ of (2.14) and (3.11).

For the discussion that follows, the equation of state relating $k_{0}$ and $\mu_{0}$ will conveniently be written as

$$
k_{0}=(\alpha-1) \mu_{0},
$$

where $\alpha$ is a parameter. ( This particular parametrisation excludes the possibility of the physically uninteresting case $\mu_{0}=0, k_{0} \neq 0$.) String matter is characterised by $\alpha=0$, whereas non-string matter has negative pressure for $0<\alpha<1$ and positive pressure if $\alpha>1$.

By definition the ring is self-supporting when no external radial stresses are necessary to prevent collapse. This happens, by virtue of (3.11), if and only if $\omega$ takes the critical value $\omega_{\text {crit }}$ that forces $C_{2}$ to vanish. Equations (3.8) and (3.10), upon inserting (5.1), imply that $\omega_{\text {crit }}$ is the solution of

$$
0=\mu_{0}\left[-1+\frac{\alpha}{1-\left(\omega_{\text {crit }} a\right)^{2}}\right] .
$$

The above constraint always has the trivial solution $\mu_{0}=0$, which in turn implies $k_{0}=0$, so that spacetime is flat everywhere. For string matter $\alpha=0$, and this trivial solution is the only solution that (5.2) admits. Consequently, we have established that a ring of string matter cannot be made self-supporting exclusively by centrifugal force but that a certain amount of external stress is necessary to prevent collapse. In other words, trying to support a ring of string matter purely by inducing a revolution requires the string to be massless, which is non physical.

The solution of $(5.2)$ in the physically interesting case, $\mu_{0} \neq 0$, is

$$
\left(\omega_{\text {crit }} a\right)^{2}=1-\alpha
$$

We observe that $\omega_{\text {crit }}$ is independent of $G$, and therefore of the gravitational field. This is a simple manifestation of the well-known [9] "Motion of the Source" problem in the linearised Einstein equations: the linear approximation is sufficient to calculate the first metric correction produced by the source but neglects the back-reaction of gravity onto the source, so that the source moves as is gravity were absent. Taking this back-reaction into 
account requires using at least second-order terms as done, for instance, in the EinsteinInfeld-Hoffman procedure [10] for the motion of point-masses. It is important, however, to insist on the fact that, although the first-order framework is not appropriate to study gravitational influences on the motion of the source, it is perfectly valid to study gravitational corrections to the metric, and thus our result on the angular deficit holds.

Further insight on the physical significance of (5.3) may be gained by studying, in Classical Mechanics, the equilibrium condition for a revolving ring of radius $a$, linear mass density $\mu$ and tension $T$. Consider a small arc of angular width $\theta$ along the circle. The two extremities of this arc are subjected to a tension $T$ which is tangential, but the resultant force $T_{R}$ at the mid-point along the arc is purely radial and is given by $T_{R}=2 T \sin (\theta / 2)$. On the other hand, the centrifugal force $F_{C}$ acting on the arc reads $F_{C}=\mu \theta \omega^{2} a^{2}$, and consequently, equilibrium is attained when $\omega$ reaches the critical value $\omega_{\text {crit }}$ satisfying

$$
\left(\omega_{\text {crit }} a\right)^{2}=\frac{T}{\mu} \lim _{\theta \rightarrow 0}[(2 / \theta) \sin (\theta / 2)]=\frac{T}{\mu}
$$

This result is identical with (5.3) for $T=-k_{0}$ and $\mu=\mu_{0}$ since $\alpha=1+k_{0} / \mu_{0}$ by (5.1).

It follows from (5.3) that matter must have a negative pressure to lead to a selfsupporting ring [since $\alpha$ must be less than one for (5.3) to admit a solution for $\omega_{\text {crit }}$ ]. This is physically reasonable since a positive pressure would tend to create an expansion of the ring, whereas a negative pressure would tend to create a collapse. Only a collapse, and therefore a negative pressure, could be counteracted by Centrifugal Force. (The gravitational force does not contribute to the collapse at first order, as just mentioned.)

Moreover, as the pressure becomes more negative and approaches $-\mu_{0}$, the critical velocity increases. The extreme case of self-supporting string matter, namely $\alpha=0$, formally implies that $\omega_{\text {crit }} a= \pm 1$, which means that the string revolves at the speed of light. This is impossible for a massive body. Thus, we reach the same conclusion as before that string matter cannot be made self-supporting by revolution only. (This may no longer hold if gravity is explicitly taken into account in the motion of the source.) The present argument, albeit physically enlightening, is only formal since the weak-field approximation breaks down for a massive body if $\omega a= \pm 1$.

We emphasise that the stress-energy tensor used in our treatment contains terms proportional to $\mu_{0}\left(1-a^{2} \omega^{2}\right)^{-1}$ and $k_{0}\left(1-a^{2} \omega^{2}\right)^{-1}$. Therefore, our results are valid when the dimensionless quantities $G \mu_{0}\left(1-a^{2} \omega^{2}\right)^{-1}$ and $G k_{0}\left(1-a^{2} \omega^{2}\right)^{-1}$ are small. In particular, the value of $\omega a$, for a ring partially supported by revolution and partially by external stresses, is not determined by any equation [in contrast with the totally self-supporting case (5.2), (5.3)], but is a free parameter. Therefore, our main result, namely that the angular deficit 
produced by a revolving ring is the same as for a straight string of the same linear density, is valid, within the confines of the weak-field limit, for a large range of the values of the parameter $a \omega$.

\section{Conclusion}

In this work, we extended our previous results [4] on the deficit-angle structure of spacetime of a non-revolving circular string to the case of a revolving circular string. The circular string was prevented from collapse partially by the centrifugal force produced by the revolution and partially by external radial stresses that were determined by stress-energy conservation. We established, in a weak-field treatment, that a conical singularity exists along the string and that the magnitude of the corresponding deficit angle is the same as that produced by a straight string of the same linear energy density.

We also investigated whether a revolving circular ring could be totally self-supporting, that is whether there existed a critical angular velocity $\omega_{\text {crit }}$ at which the ring is in equilibrium without the presence of external radial stresses. We took string matter to have the equation

of state $k_{0}=-\mu_{0}$, where $-k_{0}$ and $\mu_{0}$ denote the tension and the linear rest-energy of the string, respectively, and found that it was impossible to have a self-supporting string. However, for non-string matter characterised by the equation of state $k_{0} \neq-\mu_{0}$, the critical angular velocity was established to be

$$
\left(\omega_{\text {crit }} a\right)^{2}=-k_{0} / \mu_{0}
$$

where $a$ is the radius of the ring. [The weak-field treatment was shown to be valid as long as the dimensionless quantities $G \mu_{0}\left(1-a^{2} \omega^{2}\right)^{-1}$ and $G k_{0}\left(1-a^{2} \omega^{2}\right)^{-1}$ are small compared to unity.]

\section{Acknowledgements:}

M.V. gratefully acknowledges the Royal Irish Academy for a grant from the Research Project Development Fund and the Cork Regional Technical College (in particular Mr. R. Langford and Mr. P. Kelleher) for Leave of Absence. It is a pleasure to thank S.J. Hughes and L. O’Raifeartaigh for enlightening discussions about this problem. 


\section{Appendix A. Frame components of the Field equa- tions}

The most general stationary metric produced by an axially-symmetric source, namely (3.1), may be written in terms of an orthonormal basis, $\left\{\theta^{(\hat{\mu})}\right\}$ as

$$
d s^{2}=\eta_{\mu \nu} \theta^{(\hat{\mu})} \otimes \theta^{(\hat{\nu})}
$$

where $\eta_{\mu \nu}=\operatorname{diag}(-1,1,1,1)$ and

$$
\begin{aligned}
\theta^{(\hat{0})} \equiv e^{\nu} d t & \theta^{(\hat{1})} & \equiv r e^{\zeta-\nu}(d \phi-A d t) \\
\theta^{(\hat{2})} \equiv e^{\eta-\nu} d r & \theta^{(\hat{3})} & \equiv e^{\eta-\nu} d z .
\end{aligned}
$$

We define the transformation matrix $e^{\hat{\mu}}{ }_{\nu}$ (and its inverse $\left.e^{\mu}{ }_{\hat{\nu}}\right)$ by

$$
\theta^{(\hat{\mu})} \equiv e_{\nu}^{\hat{\mu}} d x^{\nu}
$$

The components of the Einstein tensor and the stress-energy tensor in the frame $\theta^{(\hat{\mu})}$ are then simply

$$
G_{\hat{\mu} \hat{\nu}}=G_{\alpha \beta} e_{\hat{\mu}}^{\alpha} e_{\hat{\nu}}^{\beta} \quad ; \quad T_{\hat{\mu} \hat{\nu}}=T_{\alpha \beta} e_{\hat{\mu}}^{\alpha} e_{\hat{\nu}}^{\beta},
$$

and Einstein's equations read $G_{\hat{\mu} \hat{\nu}}=-8 \pi G T_{\hat{\mu} \hat{\nu}}$.

We now turn to the weak-field approximation. The metric (A.1) is flat if $\nu=\eta=$ $\zeta=0$ and $A$ is constant. Furthermore, the quantities $\nu, \eta, \zeta$ are dimensionless, whereas $A$ has dimensions of $1 /$ length. Therefore, it is not immediately clear that a meaningful approximation to Einstein's equations can be obtained by neglecting terms of order $A^{2}$.

To clarify this point, we begin by calculating the Einstein tensor in the orthonormal frame (A.2) up to terms of first order in $\nu, \eta, \zeta$ and all orders in $A$. The results are

$$
\begin{aligned}
G_{\hat{0} \hat{0}} & =-2\left(\nabla^{2} \nu\right)+\left(\nabla^{2}+\frac{1}{r} \partial_{r}\right) \zeta+\widetilde{\nabla}^{2} \eta+\frac{r^{2}}{4}(1+2 \epsilon)\left(A_{r}^{2}+A_{z}^{2}\right) \\
G_{\hat{1} \hat{1}} & =-\widetilde{\nabla}^{2} \eta+\frac{3 r^{2}}{4}(1+2 \epsilon)\left(A_{r}^{2}+A_{z}^{2}\right) \\
G_{\hat{2} \hat{2}} & =-\zeta_{z z}-\frac{\eta_{r}}{r}+\frac{r^{2}}{4}(1+2 \epsilon)\left(A_{z}^{2}-A_{r}^{2}\right) \\
G_{\hat{3} \hat{3}} & =-\zeta_{r r}+\frac{1}{r}\left(\eta_{r}-2 \zeta_{r}\right)+\frac{r^{2}}{4}(1+2 \epsilon)\left(A_{r}^{2}-A_{z}^{2}\right) \\
G_{\hat{2} \hat{3}} & =\zeta_{r z}+\frac{1}{r}\left(\zeta_{z}-\eta_{z}\right)-\frac{r^{2}}{2}(1+2 \epsilon) A_{r} A_{z} \\
G_{\hat{0} \hat{1}} & =-\frac{r}{2} \mathcal{L} A
\end{aligned}
$$


with

$$
\mathcal{L} \equiv e^{-2(\eta+\zeta-2 \nu)}\left\{\partial_{r}\left[e^{(3 \zeta-4 \nu)} \partial_{r}\right]+\partial_{z}\left[e^{(3 \zeta-4 \nu)} \partial_{z}\right]+\frac{3}{r}\left[e^{(3 \zeta-4 \nu)} \partial_{r}\right]\right\}
$$

where $\epsilon \equiv \zeta-\nu-\eta, \widetilde{\nabla}^{2} \equiv \partial_{r}^{2}+\partial_{z}^{2}$ and $\nabla^{2} \equiv \widetilde{\nabla}^{2}+(1 / r) \partial_{r}$ is the Laplacian operator. [In equation (A.11), the exponentials are only to be taken to first order in their arguments.]

The stress-energy tensors (2.4) and (2.13) are proportional to $\mu_{0}$ and $k_{0}$, respectively, and thus, their contributions to the Einstein equations are proportional to the dimensionless quantities $G \mu_{0}$ and $G k_{0}$. Therefore, the metric appearing in the expressions for the stressenergy tensors (2.4) and (2.13) may be replaced by the flat-space metric, namely $\nu=\eta=$ $\zeta=0, A=$ const, if attention is restricted to a first-order treatment in $G \mu_{0}$ and $G k_{0}$. Hence, without making any approximation on the order of $A$, we calculate that the $T_{\hat{0} \hat{1}}$ component of the total stress-energy tensor (2.15) is

$$
G T_{\hat{0} \hat{1}}=G\left(\mu_{0}+k_{0}\right) \frac{a(A-\omega)}{1-a^{2}(A-\omega)^{2}} \delta(r-a) \delta(z)
$$

Consequently, the Einstein equation $G_{\hat{0} \hat{1}}=-8 \pi G T_{\hat{0} \hat{1}}$ becomes

$$
\mathcal{L} A=16 \pi G\left(\mu_{0}+k_{0}\right) \frac{A-\omega}{1-a^{2}(A-\omega)^{2}} \delta(r-a) \delta(z) .
$$

We now introduce the dimensionless function $B$ by

$$
A \equiv-16 \pi G\left(\mu_{0}+k_{0}\right) \omega B
$$

Note that $A$ tends to zero with $\omega$, so that the above parametrisation guarantees compatibility between the revolving case and the non-revolving case [4], where $A$ vanishes identically. Thus, the following equation for $B$ is equivalent to (A.13):

$$
\mathcal{L} B=\frac{1+16 \pi G\left(\mu_{0}+k_{0}\right) \omega B}{1-a^{2} \omega^{2}\left[1+16 \pi G\left(\mu_{0}+k_{0}\right) B\right]^{2}} \delta(r-a) \delta(z) .
$$

Expanding $B$ in powers of the dimensionless quantity $G\left(\mu_{0}+k_{0}\right)$, we clearly see from (A.15) that the leading term ${ }^{0} B$ is of zeroth order in $G\left(\mu_{0}+k_{0}\right)$ and satisfies

$$
\mathcal{L}^{0} B=\frac{1}{1-a^{2} \omega^{2}} \delta(r-a) \delta(z)
$$

As a result, by virtue of (A.14), the leading term in $A$ is proportional to $G\left(\mu_{0}+k_{0}\right)$. Therefore, we may neglect second powers of $A$ and products of $A$ with $\nu, \eta$, or $\zeta$ in our approximation scheme, since we only retain first powers of $G \mu_{0}$ and $G k_{0}$. 
*Address after September 1, 1992: Dept. of Mathematics, Statistics, and Computer

Science, Dalhousie University, Halifax, Nova Scotia, Canada B3H 4H6.

${ }^{\dagger}$ Research Associate of the Dublin Institute for Advanced Studies.

\section{References}

[1] Ya. B. Zel'dovich, Mon. Not. Roy. Astron. Soc. 192, 663 (1980); A. Vilenkin, Phys. Rep. 121, 263 (1985); R.H. Brandenberger, Physics Scripta T36, 141 (1991); A. Vilenkin, Phys. Rev. Lett. 46, 1169, 1496E (1981); A. Vilenkin, Phys. Rev. D 23, 852 (1981).

[2] A. Vilenkin, Phys. Rev. D 24, 2082 (1981); A. Vilenkin, Phys. Lett. 107B, 47 (1981); A. Vilenkin, Ap. J. 282, L51 (1984); J.R. Gott, Ap. J. 288, 422 (1985); N. Kaiser and A. Stebbins, Nature 310, 391 (1984); C.J. Hogan and M.J. Rees, Nature 311, 109 (1984); T. Vachaspati and A. Vilenkin, Phys. Rev D 31, 3052 (1985); R. Brandenberger, A. Albrecht and N. Turok, Nuc. Phys. B277, 605 (1986); F. Accetta and L. Krauss, Nuc. Phys. B319, 747 (1989); N. Sanchez and M. Signore, Phys. Lett. 219B, 413 (1989).

[3] V.P. Frolov, W. Israel, and W.G. Unruh, Phys. Rev. D 39, 1084 (1989).

[4] S.J. Hughes, D.J. Mc Manus, and M.A. Vandyck, Weak-Field Gravity of Circular Cosmic Strings, submitted to Phys. Rev. D (1992).

[5] J.L. Synge, Relativity: The General Theory (North-Holland, Amsterdam, 1960), Chap. 8, p. 310 and Chap. 4, p. 186.

[6] J. Preskill Architecture of the Fundamental Interactions at Short Distances, edited by P. Ramond and R. Stora (North Holland, Amsterdam, 1987).

[7] S. Chandrasekhar and J.L. Friedman, Astrophys. J. 175, 379 (1972).

[8] H. Bateman, Partial Differential Equations of Mathematical Physics (Cambridge Univ. Press, Cambribge, 1952), p. 461.

[9] H.C. Ohanian, Gravitation and Spacetime (Norton and co., New York, 1976), Chap. 3, p. 103.

[10] A. Einstein, L. Infeld, and B. Hoffmann, Ann. Math. 39, 65 (1938); A. Einstein and L. Infeld, Canad. J. Math. 1, 209 (1949). 